\title{
A Rapid, Proteomic Urine Assay for Monitoring Progressive Organ disease in Fabry Disease
}

§Ivan Doykov BSc ${ }^{1}$, §Wendy E. Heywood PhD ${ }^{1,2}$, Valeria Nikolaenko MSc ${ }^{1,2}$, Justyna Śpiewak MSc1 , Jenny Hällqvist BSc ${ }^{1}$, Peter Clayton MD1 Philippa Mills $\mathrm{PhD}^{1}$, David G. Warnock MD³, *Albina Nowak MD \& *\$Kevin Mills PhD ${ }^{1,2}$

1 Inborn Errors of Metabolism Section, UCL Institute of Child Health, 30 Guilford Street, London, WC1N 1EH UK

3 Division of Nephrology, Department of Medicine, University of Alabama at Birmingham, Birmingham, Alabama, USA

4 Department of Endocrinology and Clinical Nutrition, University Hospital Zurich and University of Zurich, Raemistrasse 100,CH-8091 Zurich

Authors contributed equally- §joint first authors, *joint last authors

ABSTRACT: (250 words max)

Background Fabry disease is a progressive multisystemic disease, which affects the kidney and cardiovascular systems. Various treatments exist but decisions on how and when to treat are contentious. The current marker for monitoring treatment is plasma lyso-Gb3 but it is not informative about the underlying and developing disease pathology.

Methods We have created a urine proteomic assay containing a panel of biomarkers designed to measure disease-related pathology include the inflammatory system, lysosome, heart, kidney, endothelium and cardiovascular system. Using a targeted proteomic-based approach, a series of 40 proteins for organ systems affected in Fabry Disease were multiplexed into a single 10-minute multiple reaction monitoring LCMS/MS assay and using only $1 \mathrm{ml}$ of urine.

Results Six urinary proteins were elevated in the early stage / asymptomatic Fabry group compared to controls including albumin, uromodulin, $\alpha 1$ antitrypsin, glycogen phosphorylase brain-form, endothelial protein receptor C and ICAM1. Albumin demonstrated an increase in urine and could indicate presymptomatic disease. The only protein elevated in the early stage / asymptomatic patients that continued to increase with progressive multi-organ involvement was glycogen phosphorylase brain-form. Podocalyxin, Fibroblast Growth Factor 23, Cubulin and AMBP were elevated only in disease groups involving kidney disease. Nephrin, a podocyte specific protein, was elevated in all symptomatic groups. Prosaposin was increased in all symptomatic groups and showed greater specificity $(p<0.025-0.0002)$ according to disease severity.

Conclusion This work indicates that protein biomarkers could be helpful and used in conjunction with plasma lyso-Gb3 for monitoring of therapy or disease progression in Fabry Disease patients. 


\section{Introduction}

Fabry disease (FD; Anderson-Fabry disease; OMIM \#301500) is an X-linked lysosomal storage disorder caused by mutations in the GLA gene, that leads to a deficiency of $\alpha$-galactosidase $A(\alpha-G A L){ }^{1-2}$. The consequent progressive intracellular accumulation of neutral glycosphingolipids, primarily globotriaosylceramide (Gb3, CTH or GL-3), is proposed to contribute to a wide variety of clinical symptoms of FD. In males with the Classic phenotype, who have an absent or very low $\alpha-G A L$ activity, early symptoms, including acroparathesia, episodic pain crises, angiokeratomas, hypohidrosis, gastrointestinal disturbance and corneal opacities, usually develop in early childhood and the disease gradually progresses with age, resulting in renal, cerebral and cardiac involvement ${ }^{2}$. In contrast, in males with the Later-Onset phenotype, who have a significant residual a-GAL activity, renal or cardiac involvement occur in adulthood, without the preceding early symptoms ${ }^{3-8}$. Although FD is an X-linked trait, females do exhibit disease symptoms, however this is generally in a delayed and milder form due to a random X-chromosomal deactivation ${ }^{9}$. Life expectancy in females is reported to be reduced by 15 years compared with the unaffected population, with untreated males having a median survival of 50 years ${ }^{10-11}$. The incidence of FD is estimated to range from one in 40,000 to one in $117,000^{3-8}$. However, due to the broad spectrum of clinical symptoms, many patients may remain undiagnosed $^{12}$.

Treatment for Fabry disease comprises symptomatic management, chaperone, substrate deprivation and enzyme replacement therapy (ERT). When to begin treatment of patients is often a difficult decision and, especially in children, can be a contentious issue. Additionally, there is also the dilemma of deciding when or whether to start treatment in asymptomatic patients, including some of whom may have family members who are severely affected in later life. In addition, the high expense of ERT ( $\$ 150-250 \mathrm{~K}$ per year) needs to be taken into consideration. The effectiveness of the treatment is also a debatable issue with varying studies offering different conclusions in regards to recommendations on when treatment should be started, on what dose levels and the prediction of different organs responding better to treatment than others ${ }^{13-15}$. This is compounded by conflicting views on which biomarkers are the best measure of response to treatment. Conventional biomarkers in Fabry disease include, plasma and urinary Gb3 levels ${ }^{16}$ and, more recently, plasma and urinary lyso-Gb3 levels ${ }^{6,17-18}$. Auray-Blais et al., have suggested that the measurement of analogues of lyso- Gb3 in both, plasma and urine, may be more informative in monitoring both organ involvement and response to treatment ${ }^{19-21}$.

Several studies are underway to decide on the definitive body fluid and Gb3 or lyso-Gb3 lipid moiety that determine response to treatment. In this study, we have taken an alternative approach and attempted to measure downstream markers of the disease-related pathology. Using a targeted proteomic-based test, a series of protein biomarkers for organ systems affected in FD were multiplexed into a 10 minute multiple reaction monitoring (MRM) LC-MS/MS assay using only $1 \mathrm{ml}$ of urine. Biomarkers included in the assay were determined according to previous biomarker discovery experiments in patients with Fabry disease ${ }^{22-23}$. In addition, further biomarkers were included after discussion with clinical colleagues and reviewing the literature ${ }^{24-28}$ and those proteins determined to be potentially useful for monitoring cardiovascular, renal, clotting and endothelial function ${ }^{29-37}$ (the full list of proteins quantitated in this assay are shown in supplementary data 2). The test was evaluated in urine samples from Fabry patients entering University 
Hospital Zurich and who had various stages of the disease progression associated with Fabry Disease. Here, we discuss the levels of these biomarkers and their potential to be used, alongside lyso-Gb3 and Gb3, for monitoring treatment and disease progression in Fabry Disease.

\section{Materials and methods}

Samples and disease grouping: The study was performed on samples provided by patients attending University Hospital Zurich. The study was conducted in accordance with the principles of the Helsinki Declaration and approved by the Ethics committee of the canton of Zurich, Switzerland (KEK-ZH 201700386). Informed consent for collecting clinical data, urine and blood samples for biobanking was obtained from all patients. Lyso Gb3 results were obtained from the blood but all research analyses presented in this work was performed on urine. We recruited 66 consecutive adult patients (males: $n=27(41 \%)$ ). All patients had a confirmed GLA-mutation diagnosis and presented for routine annual examinations at the University Hospital Zurich.

All patients had a multidisciplinary workup, including medical history, cardiac, renal, and neurological evaluations. Standard transthoracic 2D-echocardiography was routinely performed in all patients. LVMMI was calculated using the Devereux formula ${ }^{9}$. The occurrence of stroke or TIA (transient ischemic attack) was evaluated during annual examinations by asking the patient and/or using the medical records. For the present analyses, all clinical and routine laboratory results were extracted from the patients' medical notes. For the classification of the disease severity, the categorization "stroke" has been used for patients who fully or partly or did not recovered from stroke.

Sample collection and classification: Spot urine samples were collected from Adult Fabry patients ( $n=66$, 27 males and 39 females) and age-matched healthy adults, obtained from lab volunteers ( 5 males and 5 females). All samples were stored at $-80^{\circ} \mathrm{C}$ for a later batch analyses and had undergone 1 freeze thaw cycle prior to analysis.

Fabry patients were stratified according to symptoms: Heart involvement was defined as the presence of diastolic dysfunction and/or left ventricular hypertrophy (LVH) on echocardiography or heart MRI. Diastolic dysfunction was defined as the left atrial volume index $(L A V I)>34 \mathrm{~cm} / \mathrm{sek}$ and the mitral inflow with the E/e $<$ 12. LVH was defined as left ventricular thickness of $\geq 13 \mathrm{~mm}$. Kidney involvement was defined as protein/creatinine coefficient $>0.015 \mathrm{~g} / \mathrm{mmol}$ and/or eGFR according to CKD-Epi formula of $<90 \mathrm{ml} / \mathrm{min} / 1,73$ $\mathrm{m} 2$ Early stage Fabry patients were defined as having a proteinurina of $<0.03 \mathrm{~g} / \mathrm{mmol}$ creatinine and an eGFR $>90$. Cerebral involvement was defined as the history of stroke or trans-ischaemic attack (TIA). Sample information is supplied as supplementary data 1.

Targeted MRM LC-MS/MS Assay: Peptide selection and assessment, urine preparation, UPLC and mass spectrometry settings were performed as described previously ${ }^{38}$. Quantotypic peptides for each protein were selected using the open source online Global Proteome Machine MRM database at www.thegpm.org and each peptide homology validated by BLAST searching for their unique identity ${ }^{39}$. Custom synthesised peptides (Genscript, USA) were used to optimise peptide detection and determine the retention time and identify unequivocally the correct peptides in a urine matrix. The optimal peptide from the selection of 2 peptides/protein and two transitions was selected based on detection in urine, their quantitative response to 
increasing amount spikes into urine as determined by a standard curve and having no interfering peaks when checked with a pooled urine matrix. Parent / product ion $\mathrm{m} / \mathrm{z}$ and cone / collision energies are shown in supplementary data table 2 for the 41 proteins analysed in this study.

An intact protein of Yeast enolase from Waters MassPREPTM protein standard mix (429ng) was used as internal standard (Waters, UK) and added to $1 \mathrm{ml}$ of urine. Urines were vortexed and filtered using $3 \mathrm{kDa}$ molecular weight cut off filters (Millipore, UK). Protein digests were analysed using a Waters Aquity UPLC coupled to a Waters Xevo TQ-S mass spectrometer. Peptides were separated prior to MS detection using a CORTECS UPLC C18 + Column, $90 \AA, 1.6 \mu \mathrm{m}, 3 \mathrm{~mm}$ X $100 \mathrm{~mm}$ column attached to a C18+ VanGuard precolumn. Analysis was performed with chromatographic separation of peptides over a 10 minute reverse phase gradient. Quality Control (QC) runs of pooled urine digest were run in triplicate at the start of the run and then every 10 injections. A CV < +/-15\% for each QC was considered acceptable. Chromatograms were analysed using Waters TargetLynx software. Peptides were quantitated using the unique yeast enolase peptide (NVNDVIAPAFVK) and calibration curves generated against standard peptides for each protein (GenScript, USA). Finally, all proteins were expressed relative to urinary creatinine to correct for the strength of each urine as per normal chemical pathology procedure as described previously ${ }^{22}$. Urine creatinine was determined using mass spectrometry and as described previously by Manwaring et $\mathrm{al}^{19}{ }^{22}$ and results expressed as $\mu \mathrm{g}$ of protein / $\mathrm{mmol}$ of creatinine. A pooled sample was created using equal volumes from each sample and spiked with 1ng of synthetic peptides. This pooled QC was injected at equal intervals throughout the run to record the retention time and MS performance. Analyte/ Internal standard ratio was evaluated at $\mathrm{CV}<15 \%$.

Statistical Analysis: Data were exported to Excel. Mean values of duplicates were exported to GraphPad Prism 7 for statistical analysis. Analyses included data QC for peptide performance (coefficient of variation), QC of sample preparation and LC-MS/MS performance (yeast enolase). Standard curves were analysed by linear regression analysis and Pearson's correlation. Group comparisons were performed using non parametric Mann Whitney test.

\section{Results}

\section{Urinary Proteins elevated in early stage / asymptomatic Fabry Disease patients}

Using the targeted proteomic test developed, it was possible to identify the statistically significant elevation of 6 proteins present in the urine of genetically confirmed Fabry patients $(n=12)$, patients who demonstrated no prior symptoms of the disease i.e. no renal or cardiovascular involvement as determined by microalbuminuria, GFR or MRI imaging. All biomarkers developed into the test are shown in supplementary figure 1 and were compared to the 'gold standard' plasma lyso-Gb3 biomarker.

Figure 1 shows the protein biomarkers $\alpha_{1}$ antitrypsin (b) and uromodulin (c) demonstrated the highest significance and fold-elevation compared to age-matched controls $(p=0.0005$ and 7.7 -fold elevation, $p=0.007$ and 7.7-fold elevation, respectively). Although the three urinary proteins $\alpha_{1}$ antitrypsin, uromodulin and glycogen phosphorylase brain-form, demonstrated a higher fold-change difference in Fabry patients, they 
were not as statistically significantly elevated when compared to plasma lyso-Gb3 levels. Interestingly, urinary albumin levels were observed to be 3.1 fold elevated $(p=0.0095)$ compared to the control group and indicated the presence of subtle microalbuminuria that could not be detected using conventional routine testing techniques used in hospital laboratories. A total of $50 \%(6 / 12)$ of the patients demonstrated urinary albumin levels greater than the controls.

Although, plasma lyso-Gb3 analyses proved to be a slightly better biomarker for detecting Fabry Disease patients with little-to-no clinical symptoms than the proteins used in this study, Figure 2 shows that plasma lyso-Gb3 could not detect all Fabry Disease patients. A single female patient aged 46 carrying the R301Q mutation, not on therapy and displaying no symptoms of cardiovascular or renal involvement, demonstrated plasma lyso-Gb3 levels within the control range. This mutation has been previously reported to encode for a residual $\alpha-G A L$ activity and therefore to cause the later-onset phenotype ${ }^{41}$. However, in contrast to plasma lyso-Gb3 levels, both the urinary uromodulin and albumin levels in this patient were demonstrated to be 22 fold elevated when compared to the highest control value for uromodulin, and 1.6 fold higher than the highest albumin control. Although comprehensive gene testing, enzymology in males and possibly histological analysis of biopsies are probably the most reliable methods to diagnose Fabry Disease, this finding indicates that urinary uromodulin and albumin levels could be useful in the management of treatment patients with Fabry Disease. Both these biomarkers might also be useful in understanding the more subtle disease causing mutations as well as possibly identify the Fabry patients likely to go on to develop kidney disease.

\section{Urinary Proteins with a potential to monitor lysosomal function / hypertrophy}

The multiplexed panel used in the study included urinary proteins that were reported to be of lysosomal origin or associated with the lysosome itself. Although some overlap between the different severities of the disease was observed, in general three of these proteins prosaposin, $\mathrm{GM}_{2}$ Activator Protein and Sortilin (Figure 3a, $\mathrm{b}$ and $\mathrm{c}$, respectively) demonstrated significant changes with the progression of the disease and that potentially reflect lysosomal hypertrophy or substrate accumulation. The cohorts were grouped according to clinical pathology as described previously in the methods section. Urinary prosaposin levels demonstrated the most marked changes in progression or organ involvement of Fabry Disease (Figure 3a). Significant differences in urinary prosaposin levels were also observed between patients with no symptoms and cardiac involvement only $(p=0.0249)$, patients with renal impairment only $(p=0.0049)$, patients with renal and cardiac involvement $(p<0.001)$ and patients with a history of stroke and renal and cardiac involvement $(p=0.0002)$. Figures $3 b$ and $3 c$ show the results of the urinary levels of $\mathrm{GM}_{2}$ Activator Protein and Sortilin respectively. Although the general trend for urinary levels of both proteins showed an increase with the disease severity, observed elevated levels of $\mathrm{GM}_{2}$ Activator Protein and Sortilin did not reach statistical significance until the occurrence of marked kidney damage or when patients had a clinical history of renal, cardiac events and stroke (Figure $3 a$ and $b$ ). These data indicate that prosaposin is a potentially better biomarker of lysosomal hypertrophy or dysfunction than $\mathrm{GM}_{2}$ Activator Protein and Sortilin.

\section{Urinary Protein Biomarkers with a potential to monitor renal injury and/or impairment}

A total of six proteins known to be of kidney origin, or associated with kidney disease, demonstrated a significant change in urinary levels with progression of the clinical symptoms associated with Fabry Disease (Figure 4). The potential podocyte markers nephrin and podocalyxin both showed significant elevation with 
disease progression although this relationship dropped markedly in the more severe Fabry disease patients who exhibited a history of stroke, kidney and cardiac involvement. Podocalyxin, Fibroblast Growth Factor 23 and cubilin appear to be associated specifically with disease groups with kidney disease, as a significant difference was observed not just for the kidney involvement only and early stage/ asymptomatic Fabry groups, but also the cardiovascular manifestations only disease group.

The proteins cubilin and AMBP both showed a general increase in concentration with disease progression (Figure $4 \mathrm{e}$ and f, respectively). However, these biomarkers did not show the sensitivity of Nephrin, Podocalyxin and Fibroblast Growth Factor 23 as their increased levels were not significant from both the nonkidney disease groups until the more advanced disease groups, indicating these proteins are only altered in advanced disease.

Uromodulin levels were observed to be significantly lower in Fabry patients with both kidney and cardiac involvement $(p=0.0045)$, and in the late stage patients exhibiting kidney, heart and CNS involvement $(p=0.0049)$. Urinary albumin levels, although demonstrating statistically significant elevation in the urine of Fabry Disease patients relative to controls (figure 2), did not demonstrate any significant elevation relative to the other stages of Fabry disease (Supplementary data figure 2). Somewhat surprisingly, with the higher levels of albumin observed in patients with moderate to advanced kidney disease, these data probably arising from the sensitivity of targeted proteomics, resulted in a limited linearity range and the failing of the assay to measure the protein accurately at very high amounts (detector saturation and chromatography splitting).

\section{Urinary Protein Biomarkers with a potential to monitor cardiac involvement}

A total of 5 proteins were included in the assay for their potential to monitor cardiac disease. Lumican was identified in a previous biomarker discovery experiment as a marker of hypertrophic cardiomyopathy ${ }^{42}$ while brain natriuretic brain protein (BNP), glycogen phosphorylase B, Troponin $\mathrm{T}$ and Troponin I are known biomarkers of cardiac disease (Figure 5a-e). All 5 potential biomarkers demonstrated a general increase with the disease progression, with Glycogen Phosphorylase B and Troponin T showing a significant elevation observed in patients presenting with a cardiac phenotype only $(p=0.043$ and 0.036 , Figure $5 b$ and $5 c$, respectively). None of the other cardiac proteins were significantly elevated in the cardiovascular only group compared to the early stage / asymptomatic group. However, significantly elevated and higher levels of all the proteins were also observed in patients presenting with kidney disease and no significant cardiac involvement. These data indicate that the cardiac biomarkers are potentially useful for identifying progressive but not early cardiac involvement and their levels are probably confounded by the later involvement of kidney disease. However, these levels could reflect that cardio-renal syndrome which is a very complex scenario and drawing conclusions are really a challenge in the sample set used in this work. Further research on more and well characterised patients with cardiovascular and kidney disease would need to be undertaken to better understand the significance of these results.

\section{Potential inflammatory protein biomarkers present in the urine}

The inflammatory proteins neutrophil collagenase, hemopexin, intracellular adhesion molecule 1 and $\alpha_{1}$. antitrypsin (Figure 6 a-d) demonstrated elevated levels in the majority of patients with Fabry disease. However, this was not observed in all cases and some overlap between the different disease stage groups 
were observed. The inflammatory markers neutrophil collagenase and hemopexin both demonstrated significantly elevated levels that corresponded with disease progression and indicating that inflammation may play an important role in the disease pathogenesis observed with Fabry Disease (Figure 6a and b). Intracellular adhesion molecule 1, although elevated in the cardiac, kidney, cardiac and kidney, and those patients with cardiac, kidney and CNS involvement, the levels in the urine only reached significant elevation in patients with kidney disease only $(p=0.0031)$. Although $\alpha_{1}$-antitrypsin demonstrated significant and almost 8-fold elevated levels between controls and Fabry patients exhibiting no symptoms clinically (Figure 1a), these levels did not rise significantly with further progression of the clinical symptoms observed in Fabry Disease (Figure 6d). 


\section{Discussion}

The main aim of this proof of principle project was to see if we could develop a non-invasive, multiplexed and targeted proteomic method that could supplement the conventional tests used to aid diagnosis and monitor treatment for Fabry Disease. In-depth testing of such biomarkers would aid in development of emerging and novel therapies. In total, a method was developed to detect 41 proteins in $1 \mathrm{ml}$ of urine (listed in Supplementary data table 2). All 41 proteins from this panel could be reliably quantitated but only 20 biomarkers showed any potential changes and use for the diagnosis and monitoring of disease progression in Fabry disease. The biomarkers could be divided into 2-groups, those that had potential for aiding the diagnosis of Fabry Disease and those that could be used potentially for monitoring treatment or studying disease progression.

Surprisingly, the assay proved useful for looking at the early stage / presymptomatic or early stages of disease as opposed to the later stages of Fabry Disease, when patients develop a more severe phenotype (kidney, cardiac and CNS involvement or stroke). An interesting finding was the presence of specific proteins in the urine of patients who presented with no symptoms of Fabry Disease using conventional tests (MRI, GFR, microalbuminuria and plasma lyso-Gb3). $\alpha_{1}$-Antitrypsin and uromodulin both showed significantly elevated levels in the urine of the pre-symptomatic patients. Although not as useful as plasma lyso-Gb3 for diagnosing Fabry Disease, both demonstrated a greater fold elevation ( 7.7-fold) compared to plasma lyso-Gb3 ( 6fold elevation). The reasons for this elevation of $\alpha_{1}$ antitrypsin in the urine are unclear but have been reported previously by Maicas et al ${ }^{43}$. They postulated that the elevation of $\alpha_{1}$ antitrypsin maybe an anti-inflammatory or cytoprotective effect while other studies ${ }^{44-46}$ demonstrated that $\alpha_{1}$ antitrypsin levels could be used to predict and monitor kidney function in type-2 diabetic nephropathy, glomerulonephritis and monitoring kidney disease, respectively. Similarly, the elevation of uromodulin, or Tamm Horsfall protein, has been described previously as a good biomarker for quantitating kidney function in Fabry Disease ${ }^{47}$ and as a potent immunomodulatory molecule in kidney disease ${ }^{48}$. The latter research agrees with our results that the elevation of $\alpha_{1}$ antitrypsin indicated an inflammatory component, or involvement, in the early stages of Fabry Disease. Other proteins showing an elevation in the early stage / presymptomatic patients included glycogen phosphorylase B (GPB), albumin, endothelial receptor C and podocalyxin. GPB is highly expressed in the brain and the heart and was included in the panel as a potential biomarker of heart disease. Previous studies 4950 have shown that GPB is a good marker of acute myocardial injury or infarction. Although acute cardiac injury is not a hallmark of Fabry Disease, GPB demonstrated both, a significant fold-change elevation (6-fold) and indicated that it could be a potential and subtle early marker of cardiac disease in early stage / presymptomatic patients. Indeed, increased levels of GPB were observed in $50 \%$ of the patients with no signs of cardiac disease (pre-symptomatic group). Surprisingly, increased urinary albumin levels indicated that it was a good protein to accompany the diagnosis of Fabry Disease with almost $50 \%$ of the pre-symptomatic patient group showing an elevation compared to controls. None of the patients were reported as having microalbuminuria using conventional tests and indicating that targeted proteomics is a much more sensitive and accurate methodology for quantitating proteins than conventional chemical laboratory assays. However, one of the limitations of the assay we found was in its inability to reliably quantitate high levels of albumin, as 
the method was designed to look only low abundant biomarkers. Further refinement of the assay will be necessary if the assay is to be used to quantitate macro albuminuria.

Although no proteins analysed in this study proved quite as specific as plasma lyso-Gb3, Figure 2 shows one patient with a confirmed later-onset mutation in the alpha galactosidase gene (female, R301Q, aged 46), who did not have an elevated plasma lyso-Gb3 level but did demonstrate significantly elevated uromodulin and albumin levels in the urine. The presence of these proteins in the urine indicates that they may be very sensitive markers of early stage / pre-symptomatic kidney disease. Although only one patient, this finding indicates that although plasma lyso-Gb3 remains helpful for diagnosing Fabry disease, it is not perfect and the use of other markers such as urinary uromodulin and albumin may help the diagnosis in difficult cases and reduce the number of false negatives when used in conjunction with plasma lyso-Gb3.

\section{Analysis of those biomarkers that may reflect disease progression}

During the development of the assay, the proteins included in the test were chosen from either preliminary proteomics experiments or thought to reflect certain organ or cellular systems known to be affected in Fabry Disease (Supplementary table 2). The lysosomal proteins that could be detected in the urine included prosaposin, $\mathrm{GM}_{2}$ activator protein and sortilin. All three proteins demonstrated a general increase in levels detected in the urine that reflected the progression of the disease and probably resulting from lysosomal hypertrophy due to storage burden. These data correlated with our previous work showing the increased levels of these biomarkers in early stage / pre-symptomatic patients ${ }^{22}$. The kidney contains very high levels of lysosomes making urine a very good fluid for lysosomal research. This may also explain the higher levels of lysosomal proteins in the urine of Fabry patients with kidney disease only compared to those with cardiac manifestations only. However, in those patients with both, kidney and heart involvement, the levels were significantly higher than individual systems alone. The highest levels of all three biomarkers were observed in the urine of patients with cardiac, kidney and some history of stroke. This is indicative of the storage burden and hypertrophy known to occur in the later stages of Fabry Disease.

Our next panel of proteins were selected to see if it was possible to quantitate kidney function and included markers of podocyte loss ${ }^{51-52}$, tubular dysfunction ${ }^{34}$ and those proteins that act on the function of the kidney. It is interesting to observe that nephrin, a specific podocyte marker, demonstrated significantly elevated levels (Figure 4a) even in those Fabry Disease patients presenting with cardiac symptoms only. This indicates, nephrin maybe a sensitive marker of early podocyte loss and therefore early stage / presymptomatic kidney disease in Fabry Disease patients who are thought to have only cardiac involvement. Podocalyxin, Fibroblast Growth factor $23^{53}$, cubilin and AMBP were not elevated in the group with only cardiovascular disease but were elevated in the kidney disease only group indicating these are markers of kidney disease. Podocalyxin levels appear to become very varied and non-specific in the other 2 groups with more multi-systemic disease. The reason for this is unclear but maybe explained by a more extensive loss of podocytes at the early stages of the disease which could be an indication of podocyte loss in these groups where disease is more advanced. Fibroblast Growth factor 23 continued to remain elevated in these groups and cubilin appeared to show a progressive increase as the disease involved more organ systems. 
Although other kidney disease associated proteins showed a similar and general pattern of increased levels observed in the urine which corresponded with kidney disease, this was not observed with uromodulin and demonstrated the inverse with Fabry Disease progression. Uromodulin is secreted by the thick ascending limb of the loops of Henle and thus the lower levels observed in the urine as the disease progresses, probably reflects tubular damage. Uromodulin is continually secreted into the urine and is thought to be an inhibitor of calcium crystallisation but may also have an antibacterial role and protection against urinary tract infections. Diseases associated with uromodulin include Hyperuricemic Nephropathy, Familial Juvenile, 1 and Medullary Cystic Kidney Disease $2^{48}$. Thus it is highly likely that the loss of uromodulin excretion in the kidney reflects kidney disease associated with Fabry Disease but may also play a part of the disease mechanism or process observed in the disease.

Interestingly, 5 proteins were selected for their potential as biomarkers capable of monitoring cardiac function and could be detected in the urine, in both, controls and Fabry Disease patients. Some of these proteins have been detected in urine before and explored for their potential utility in cardiac disease ${ }^{54-55}$. Like other biomarkers analysed in this study, the levels of all 5 proteins followed a similar pattern that corresponded with the disease progression associated with Fabry disease and, in the case of GPB and Troponin T, were significantly elevated in the patients with cardiac involvement compared to the patients with none (figure 5). However, increased levels were also observed in patients with kidney disease involvement only. This indicated that the specificity of these cardiac markers in the urine are not as specific as would have been predicted or that patients with kidney disease, allow these proteins to enter the urine more freely and thus compromised the clinical use or relevance of the markers. The reason for this discrepancy is unclear and the origin of these markers should be further elucidated by looking at different acute and chronic cardiac diseases such as myocarditis, heart failure or acute infarct damage.

Finally, the proteins neutrophil collagenase, hemopexin, ICAM1 and $\alpha_{1}$ antitrypsin, that are known to be associated with inflammation, all demonstrated significant elevation and correlated with the disease progression associated with Fabry disease. $\alpha_{1}$ Antitrypsin demonstrating a significant 7.7 fold elevation between controls and those Fabry Disease patients that did not show any symptoms of Fabry disease. However, the proteins neutrophil collagenase, hemopexin and, to a lesser extent ICAM1, all followed the disease progression. Only $\alpha_{1}$ antitrypsin did not show as much potential for monitoring inflammation as levels were not observed to follow the progression of disease as closely. Neutrophil collagenase or MMP8, is a metalloprotease secreted by neutrophils and is involved in the degradation of the extracellular matrix. It is also known to be involved in the remodelling of tissue, a hallmark of HCM and the kidney disease associated with Fabry disease. Another potential marker and indicator of an inflammatory component to Fabry disease was the protein hemopexin. Hemopexin is a heme or iron scavenging protein whose function is to protect the body from the oxidative damage and pro-inflammatory nature of iron in the circulation. Hemopexin has been shown to play a role in Lupus, malaria and also a potent antioxidant role in the heart ${ }^{56}$. Although hemopexin levels observed in the urine do reflect the disease progression observed in Fabry Disease, what exactly they are indicative of is unclear and could reflect changes in iron homeostasis, inflammation, kidney or heart failure. The higher levels of pro-inflammatory proteins in the urine certainly indicate that oxidative stress or inflammation may play a part in Fabry Disease. 
All patients who were symptomatic in this study were on ERT therefore the proper assessment of these markers in response to ERT was not possible. A longitudinal study is required to ascertain this. The effect of ERT could explain the widespread heterogeneity of some of the protein values observed in the disease groups (particularly more so in the later stage disease groups) as there may be some patients responding or not responding to the ERT. Plasma lyso-Gb3 is useful for determining disease progression in male Fabry patients ${ }^{57}$ but when grouped according to disease symptoms in this cohort, similarly to the protein markers quantitated in this study, there was no significant differences in the lyso-Gb3 values observed according to disease (supplementary data figure 3). The inclusion of hemizygous females in the cohort who typically have lower Gb3 and lyso-Gb3 levels, may explain why a relationship to disease was not observed for lyso-Gb3. We also looked at any possible relationship of lyso-Gb3 with any of the proteins by Spearman multiple correlation analysis (supplementary data figure 4). No correlation of plasma lyso-Gb3 with any of the proteins was observed, indicating their expression is independent of lyso-Gb3 and is more likely related to the specific organ pathology of the disease. However, a Spearman multiple correlation analysis of Fabry patients demonstrated a strong negative correlation between most proteins and uromodulin. In particular, a strong negative correlation between uromodulin and $\mathrm{GM}_{2}$ activator Protein, prosaposin, Nephrin, Troponin I and Lumican were observed. All these proteins that are highly expressed in the lysosome, kidney and heart and all organs known to be affected in Fabry Disease. In contrast, a strong positive correlation was observed between Cubilin with Sortilin, Lumican and Glycogen Phosphorylase B, Troponin T with Glycogen Phosphorylase B, and finally, uromodulin with Endothelial Protein Receptor C.

The limitations of this study are the biomarkers developed into the test described in this work have only been measured in the urine of patients with Fabry disease and a significant number of patients were on treatment. The levels of these biomarkers in other patients with LSD, or other non-lysosomal causes of cardiovascular and kidney disease, might provide further insight into the relevance of these biomarkers for monitoring disease progression and treatment for Fabry Disease. In addition, a similar follow up study needs to be conducted with a well characterised and longitudinal cohort of samples, ideally from patients pre- and - posttreatment and preferably over several years.

\section{Conclusions}

We believe this rapid multiplex urine assay has much potential and indicate some proteins may be specific disease biomarkers that could have promise for monitoring treatment or disease progression.

\section{Supporting Information}

Supplementary table 1: Spreadsheet of sample information

Supplementary table 2: Table of peptide sequences and transitions

Supplementary data figure 1: UPLC-MS/MS chromatogram showing the multiplex analysis of all biomarkers quantitated in this study.

Supplementary data figures 2: Urinary albumin levels in Fabry disease grouped according to symptomatic disease

Supplementary data figures 3: Plasma Lyso-Gb3 levels grouped according to disease

Supplementary data figures 4: Spearman correlation matrix of protein levels and plasma lyso-Gb3 


\section{AUTHOR INFORMATION}

Corresponding Author

Tel: 02079052873. Fax: 02074046191 E-mail: kevin.mills@ucl.ac.uk

Author Contributions

The manuscript was written through contributions of all authors. All authors have given approval to the final version of the manuscript. The authors declare no competing interests

\section{ACKNOWLEDGMENT}

This work is supported by the NIHR GOSH BRC. The views expressed are those of the author(s) and not necessarily those of the NHS, the NIHR or the Department of Health. UCL Biological Mass Spectrometry Centre at the UCL Institute of Child Health and with kind donations from the Szeban Peto Foundation. We also wish to thank our technician $\mathrm{Mr}$ Ernestas Sirka for making up solutions.

\section{REFERENCES}

1. Bishop, D. F.; Kornreich, R.; Desnick, R. J., Structural organization of the human alpha-galactosidase A gene: further evidence for the absence of a 3' untranslated region. Proc Natl Acad Sci U S A 1988, 85 (11), 3903-7.

2. Zarate, Y. A.; Hopkin, R. J., Fabry's disease. Lancet 2008, 372 (9647), 1427-35.

3. Desnick, R.; loannou, Y.; Eng, C., $\alpha$-galactosidase A deficiency: Fabry disease. The metabolic and molecular bases of inherited disease. (eds Scriver CR, Beaudet AL, Sly WS, Valle D.) p3733-3774. McGraw-Hill, New York: 2001.

4. Nowak, A.; Mechtler, T. P.; Hornemann, T.; Gawinecka, J.; Theswet, E.; Hilz, M. J.; Kasper, D. C., Genotype, phenotype and disease severity reflected by serum LysoGb3 levels in patients with Fabry disease. Molecular genetics and metabolism 2018, Feb;123(2):148-153.

5. von Scheidt, W.; Eng, C. M.; Fitzmaurice, T. F.; Erdmann, E.; Hubner, G.; Olsen, E. G.; Christomanou, H.; Kandolf, R.; Bishop, D. F.; Desnick, R. J., An atypical variant of Fabry's disease with manifestations confined to the myocardium. The New England journal of medicine 1991, 324 (6), 395-9.

6. $\quad$ Arends, M.; Wanner, C.; Hughes, D.; Mehta, A.; Oder, D.; Watkinson, O. T.; Elliott, P. M.; Linthorst, G. E.; Wijburg, F. A.; Biegstraaten, M.; Hollak, C. E., Characterization of Classical and Nonclassical Fabry Disease: A Multicenter Study. J Am Soc Nephrol 2017, May;28(5):1631-1641.

7. Echevarria, L.; Benistan, K.; Toussaint, A.; Dubourg, O.; Hagege, A. A.; Eladari, D.; Jabbour, F.; Beldjord, C.; De Mazancourt, P.; Germain, D. P., X-chromosome inactivation in female patients with Fabry disease. Clin Genet 2016, 89 (1), 44-54.

8. Meikle, P. J.; Hopwood, J. J.; Clague, A. E.; Carey, W. F., Prevalence of lysosomal storage disorders. JAMA 1999, $281(3), 249-54$.

9. Devereux, R. B.; Reichek, N., Echocardiographic determination of left ventricular mass in man. Anatomic validation of the method. Circulation 1977, 55 (4), 613-8.

10. Tanaka, A.; Takeda, T.; Hoshina, T.; Fukai, K.; Yamano, T., Enzyme replacement therapy in a patient with Fabry disease and the development of IgE antibodies against agalsidase beta but not agalsidase alpha. $J$ Inherit Metab Dis 2010, 33 Suppl 3, S249-52.

11. MacDermot, K. D.; Holmes, A.; Miners, A. H., Anderson-Fabry disease: clinical manifestations and impact of disease in a cohort of 98 hemizygous males. Journal of medical genetics 2001, 38 (11), 750-60.

12. Doheny, D.; Srinivasan, R.; Pagant, S.; Chen, B.; Yasuda, M.; Desnick, R. J., Fabry Disease: prevalence of affected males and heterozygotes with pathogenic GLA mutations identified by screening renal, cardiac and stroke clinics, 19952017. Journal of medical genetics 2018, 55 (4), 261-268.

13. Biegstraaten, M.; Arngrimsson, R.; Barbey, F.; Boks, L.; Cecchi, F.; Deegan, P. B.; Feldt-Rasmussen, U.; Geberhiwot, T.; Germain, D. P.; Hendriksz, C.; Hughes, D. A.; Kantola, I.; Karabul, N.; Lavery, C.; Linthorst, G. E.; Mehta, A.; van de Mheen, E.; Oliveira, J. P.; Parini, R.; Ramaswami, U.; Rudnicki, M.; Serra, A.; Sommer, C.; Sunder-Plassmann, G.; Svarstad, E.; Sweeb, A.; Terryn, W.; Tylki-Szymanska, A.; Tondel, C.; Vujkovac, B.; Weidemann, F.; Wijburg, F. A.; Woolfson, P.; Hollak, C. E., Recommendations for initiation and cessation of enzyme replacement therapy in patients with Fabry disease: the European Fabry Working Group consensus document. Orphanet journal of rare diseases 2015, 10,36 . 
14. Schiffmann, R.; Swift, C.; Wang, X.; Blankenship, D.; Ries, M., A prospective 10-year study of individualized, intensified enzyme replacement therapy in advanced Fabry disease. J Inherit Metab Dis 2015, 38 (6), 1129-36.

15. Hopkin, R. J.; Jefferies, J. L.; Laney, D. A.; Lawson, V. H.; Mauer, M.; Taylor, M. R.; Wilcox, W. R.; Fabry Pediatric Expert, P., The management and treatment of children with Fabry disease: A United States-based perspective. Molecular genetics and metabolism 2016, Feb;117(2):104-13.

16. Young, E.; Mills, K.; Morris, P.; Vellodi, A.; Lee, P.; Waldek, S.; Winchester, B., Is globotriaosylceramide a useful biomarker in Fabry disease? Acta paediatrica 2005, 94 (447), 51-4.

17. Auray-Blais, C.; Ntwari, A.; Clarke, J. T.; Warnock, D. G.; Oliveira, J. P.; Young, S. P.; Millington, D. S.; Bichet, D. G.; Sirrs, S.; West, M. L.; Casey, R.; Hwu, W. L.; Keutzer, J. M.; Zhang, X. K.; Gagnon, R., How well does urinary lyso-Gb3 function as a biomarker in Fabry disease? Clinica chimica acta; international journal of clinical chemistry 2010, 411 (2324), 1906-14.

18. Boutin, M.; Gagnon, R.; Lavoie, P.; Auray-Blais, C., LC-MS/MS analysis of plasma lyso-Gb3 in Fabry disease. Clinica chimica acta; international journal of clinical chemistry 2012, 414, 273-80.

19. Manwaring, V.; Boutin, M.; Auray-Blais, C., A metabolomic study to identify new globotriaosylceramiderelated biomarkers in the plasma of Fabry disease patients. Analytical chemistry 2013, 85 (19), 9039-48.

20. Abaoui, M.; Boutin, M.; Lavoie, P.; Auray-Blais, C., Tandem mass spectrometry multiplex analysis of methylated and non-methylated urinary Gb isoforms in Fabry disease patients. Clin Chim Acta 2016, Jan 15;452:191-8.

21. Boutin, M.; Auray-Blais, C., Multiplex tandem mass spectrometry analysis of novel plasma lyso-Gb(3)-related analogues in Fabry disease. Analytical chemistry 2014, 86 (7), 3476-83.

22. Manwaring, V.; Heywood, W. E.; Clayton, R.; Lachmann, R. H.; Keutzer, J.; Hindmarsh, P.; Winchester, B.; Heales, S.; Mills, K., The identification of new biomarkers for identifying and monitoring kidney disease and their translation into a rapid mass spectrometry-based test: evidence of presymptomatic kidney disease in pediatric Fabry and type-I diabetic patients. Journal of proteome research 2013, 12 (5), 2013-21.

23. Coats, C. J.; Heywood, W. E.; Mills, K.; Elliott, P. M., Current applications of biomarkers in cardiomyopathies. Expert review of cardiovascular therapy 2015, 13 (7), 825-37.

24. Shen, J. S.; Meng, X. L.; Schiffmann, R.; Brady, R. O.; Kaneski, C. R., Establishment and characterization of Fabry disease endothelial cells with an extended lifespan. Molecular genetics and metabolism 2007, 92 (1-2), 137-44.

25. Christensen, E. I.; Gburek, J., Protein reabsorption in renal proximal tubule-function and dysfunction in kidney pathophysiology. Pediatric nephrology 2004, 19 (7), 714-21.

26. Prabakaran, T.; Nielsen, R.; Satchell, S. C.; Mathieson, P. W.; Feldt-Rasmussen, U.; Sorensen, S. S.; Christensen, E. I., Mannose 6-phosphate receptor and sortilin mediated endocytosis of alpha-galactosidase A in kidney endothelial cells. PloS one 2012, 7 (6), e39975.

27. Tan, K.; Sethi, S. K., Biomarkers in cardiorenal syndromes. Translational research : the journal of laboratory and clinical medicine 2014, 164 (2), 122-34.

28. Valino-Rivas, L.; Baeza-Bermejillo, C.; Gonzalez-Lafuente, L.; Sanz, A. B.; Ortiz, A.; Sanchez-Nino, M. D., CD74 in Kidney Disease. Frontiers in immunology 2015, 6, 483.

29. Hara, M.; Yanagihara, T.; Kihara, I.; Higashi, K.; Fujimoto, K.; Kajita, T., Apical cell membranes are shed into urine from injured podocytes: a novel phenomenon of podocyte injury. Journal of the American Society of Nephrology : JASN 2005, 16 (2), 408-16.

30. Vinken, P.; Starckx, S.; Barale-Thomas, E.; Looszova, A.; Sonee, M.; Goeminne, N.; Versmissen, L.; Buyens, K.; Lampo, A., Tissue Kim-1 and urinary clusterin as early indicators of cisplatin-induced acute kidney injury in rats. Toxicologic pathology 2012, 40 (7), 1049-62.

31. Vivekanandan-Giri, A.; Slocum, J. L.; Buller, C. L.; Basrur, V.; Ju, W.; Pop-Busui, R.; Lubman, D. M.; Kretzler, M.; Pennathur, S., Urine glycoprotein profile reveals novel markers for chronic kidney disease. International journal of proteomics 2011, 2011;2011:214715.

32. Najafian, B.; Mauer, M.; Hopkin, R. J.; Svarstad, E., Renal complications of Fabry disease in children. Pediatric nephrology 2013, 28 (5), 679-87.

33. Du, T. Y.; Luo, H. M.; Qin, H. C.; Wang, F.; Wang, Q.; Xiang, Y.; Zhang, Y., Circulating serum trefoil factor 3 (TFF3) is dramatically increased in chronic kidney disease. PloS one 2013, 8 (11), e80271.

34. Yu, Y.; Jin, H.; Holder, D.; Ozer, J. S.; Villarreal, S.; Shughrue, P.; Shi, S.; Figueroa, D. J.; Clouse, H.; Su, M.; Muniappa, N.; Troth, S. P.; Bailey, W.; Seng, J.; Aslamkhan, A. G.; Thudium, D.; Sistare, F. D.; Gerhold, D. L., Urinary biomarkers trefoil factor 3 and albumin enable early detection of kidney tubular injury. Nature biotechnology 2010, 28 (5), 470-7.

35. Takahashi, N.; Yokoi, S.; Kasuno, K.; Kogami, A.; Tsukimura, T.; Togawa, T.; Saito, S.; Ohno, K.; Hara, M.; Kurosawa, H.; Hirayama, Y.; Kurose, T.; Yokoyama, Y.; Mikami, D.; Kimura, H.; Naiki, H.; Sakuraba, H.; Iwano, M., A heterozygous female with Fabry disease due to a novel alpha-galactosidase $A$ mutation exhibits a unique synaptopodin distribution in vacuolated podocytes. Clinical nephrology 2015, 83 (5), 301-8. 
36. Suresh, C. P.; Saha, A.; Kaur, M.; Kumar, R.; Dubey, N. K.; Basak, T.; Tanwar, V. S.; Bhardwaj, G.; Sengupta, S.; Batra, V. V.; Upadhyay, A. D., Differentially expressed urinary biomarkers in children with idiopathic nephrotic syndrome. Clin Exp Nephrol. 2016, Apr;20(2):273-83

37. Tian, J.; Wang, H. P.; Mao, Y. Y.; Jin, J.; Chen, J. H., Reduced glomerular epithelial protein 1 expression and podocyte injury in immunoglobulin A nephropathy. The Journal of international medical research 2007, 35 (3), 338-45. 38. Heywood, W. E.; Camuzeaux, S.; Doykov, I.; Patel, N.; Preece, R. L.; Footitt, E.; Cleary, M.; Clayton, P.; Grunewald, S.; Abulhoul, L.; Chakrapani, A.; Sebire, N. J.; Hindmarsh, P.; de Koning, T. J.; Heales, S.; Burke, D.; Gissen, P.; Mills, K., Proteomic Discovery and Development of a Multiplexed Targeted MRM-LC-MS/MS Assay for Urine Biomarkers of Extracellular Matrix Disruption in Mucopolysaccharidoses I, II, and VI. Analytical chemistry 2015, Dec 15;87(24):12238-44

39. Craig, R.; Cortens, J. P.; Beavis, R. C., Open source system for analyzing, validating, and storing protein identification data. Journal of proteome research 2004, 3 (6), 1234-42.

40. Mills, K.; Morris, P.; Lee, P.; Vellodi, A.; Waldek, S.; Young, E.; Winchester, B., Measurement of urinary CDH and $\mathrm{CTH}$ by tandem mass spectrometry in patients hemizygous and heterozygous for Fabry disease. $J$ Inherit Metab Dis 2005, 28 (1), 35-48.

41. Kase, R.; Bierfreund, U.; Klein, A.; Kolter, T.; Utsumi, K.; Itoha, K.; Sandhoff, K.; Sakuraba, H., Characterization of two alpha-galactosidase mutants (Q279E and R301Q) found in an atypical variant of Fabry disease. Biochimica et biophysica acta 2000, 1501 (2-3), 227-35.

42. Coats, C. J.; Heywood, W. E.; Virasami, A.; Ashrafi, N.; Syrris, P.; Dos Remedios, C.; Treibel, T. A.; Moon, J. C.; Lopes, L. R.; McGregor, C. G. A.; Ashworth, M.; Sebire, N. J.; McKenna, W. J.; Mills, K.; Elliott, P. M., Proteomic Analysis of the Myocardium in Hypertrophic Obstructive Cardiomyopathy. Circ Genom Precis Med 2018, 11 (12), e001974.

43. Maicas, N.; van der Vlag, J.; Bublitz, J.; Florquin, S.; Bakker-van Bebber, M.; Dinarello, C. A.; Verweij, V.; Masereeuw, R.; Joosten, L. A.; Hilbrands, L. B., Human Alpha-1-Antitrypsin (hAAT) therapy reduces renal dysfunction and acute tubular necrosis in a murine model of bilateral kidney ischemia-reperfusion injury. PloS one 2017, 12 (2), e0168981.

44. Guo, Z.; Liu, X.; Li, M.; Shao, C.; Tao, J.; Sun, W.; Li, M., Differential urinary glycoproteome analysis of type 2 diabetic nephropathy using 2D-LC-MS/MS and iTRAQ quantification. Journal of translational medicine 2015, 13 (1), 371.

45. Smith, A.; L'Imperio, V.; De Sio, G.; Ferrario, F.; Scalia, C.; Dell'Antonio, G.; Pieruzzi, F.; Pontillo, C.; Filip, S.; Markoska, K.; Granata, A.; Spasovski, G.; Jankowski, J.; Capasso, G.; Pagni, F.; Magni, F., alpha-1-antitrypsin detected by MALDI-Imaging in the study of glomerulonephritis: its relevance in chronic kidney disease progression. Proteomics 2016, Jun;16(11-12):1759-66

46. Magalhaes, P.; Mischak, H.; Zurbig, P., Urinary proteomics using capillary electrophoresis coupled to mass spectrometry for diagnosis and prognosis in kidney diseases. Curr Opin Nephrol Hypertens 2016, 25 (6), 494-501.

47. Matafora, V.; Cuccurullo, M.; Beneduci, A.; Petrazzuolo, O.; Simeone, A.; Anastasio, P.; Mignani, R.; Feriozzi, S.; Pisani, A.; Comotti, C.; Bachi, A.; Capasso, G., Early markers of Fabry disease revealed by proteomics. Molecular bioSystems 2015, $11(6), 1543-51$.

48. Wu, T. H.; Li, K. J.; Yu, C. L.; Tsai, C. Y., Tamm-Horsfall Protein is a Potent Immunomodulatory Molecule and a Disease Biomarker in the Urinary System. Molecules 2018, 23 (1).

49. Peetz, D.; Post, F.; Schinzel, H.; Schweigert, R.; Schollmayer, C.; Steinbach, K.; Dati, F.; Noll, F.; Lackner, K. J., Glycogen phosphorylase BB in acute coronary syndromes. Clinical chemistry and laboratory medicine : CCLM / FESCC 2005, 43 (12), 1351-8.

50. Krause, E. G.; Rabitzsch, G.; Noll, F.; Mair, J.; Puschendorf, B., Glycogen phosphorylase isoenzyme BB in diagnosis of myocardial ischaemic injury and infarction. Mol Cell Biochem 1996, 160-161, 289-95.

51. Biarc, J.; Simon, R.; Fonbonne, C.; Leonard, J. F.; Gautier, J. C.; Pasquier, O.; Lemoine, J.; Salvador, A., Absolute quantification of podocalyxin, a potential biomarker of glomerular injury in human urine, by liquid chromatographymass spectrometry. Journal of chromatography. A 2015, 1397, 81-5.

52. Lioudaki, E.; Stylianou, K. G.; Petrakis, I.; Kokologiannakis, G.; Passam, A.; Mikhailidis, D. P.; Daphnis, E. K.; Ganotakis, E. S., Increased Urinary Excretion of Podocyte Markers in Normoalbuminuric Patients with Diabetes. Nephron 2015, 131 (1), 34-42.

53. Fliser, D.; Kollerits, B.; Neyer, U.; Ankerst, D. P.; Lhotta, K.; Lingenhel, A.; Ritz, E.; Kronenberg, F.; Group, M. S.; Kuen, E.; Konig, P.; Kraatz, G.; Mann, J. F.; Muller, G. A.; Kohler, H.; Riegler, P., Fibroblast growth factor 23 (FGF23) predicts progression of chronic kidney disease: the Mild to Moderate Kidney Disease (MMKD) Study. Journal of the American Society of Nephrology : JASN 2007, 18 (9), 2600-8.

54. Cortes, R.; Rivera, M.; Salvador, A.; Garcia de Burgos, F.; Bertomeu, V.; Rosello-Lleti, E.; Martinez-Dolz, L.; Paya, R.; Almenar, L.; Portoles, M., Urinary B-type natriuretic peptide levels in the diagnosis and prognosis of heart failure. Journal of cardiac failure 2007, 13 (7), 549-55. 
55. Linssen, G. C.; Damman, K.; Hillege, H. L.; Navis, G.; van Veldhuisen, D. J.; Voors, A. A., Urinary N-terminal prohormone brain natriuretic peptide excretion in patients with chronic heart failure. Circulation 2009, 120 (1), 35-41. 56. Ingoglia, G.; Sag, C. M.; Rex, N.; De Franceschi, L.; Vinchi, F.; Cimino, J.; Petrillo, S.; Wagner, S.; Kreitmeier, K.; Silengo, L.; Altruda, F.; Maier, L. S.; Hirsch, E.; Ghigo, A.; Tolosano, E., Data demonstrating the anti-oxidant role of hemopexin in the heart. Data Brief 2017, 13, 69-76.

57. Nowak, A.; Mechtler, T. P.; Hornemann, T.; Gawinecka, J.; Theswet, E.; Hilz, M. J.; Kasper, D. C., Genotype, phenotype and disease severity reflected by serum LysoGb3 levels in patients with Fabry disease. Molecular genetics and metabolism 2018, 123 (2), 148-153. 


\section{Legends to Figures}

Figure 1 Potential early stage / presymptomatic biomarkers of Fabry Disease $(n=12)$ that are present in the urine of patients that demonstrate no clinical symptoms of cardiovascular or renal impairment. The dotted line indicates the control patient upper levels.

Figure 2 Uromodulin and albumin levels in a Fabry Disease patient who has normal plasma lyso Gb3 levels and no signs of cardiovascular or renal involvement (male, aged 46 years old, R301Q mutation). The dotted line indicates the control patient upper levels.

Figure 3 Analysis of potential lysosomal markers prosaposin (a), GM1 activator protein (b) and sortillin (c) in the urine of patients with Fabry disease $(n=66)$ and their levels categorised according to disease progression. The dotted line indicates the control patient upper levels.

Figure 4 Analysis of potential kidney disease markers nephrin (a), podocalyxin (b), uromodulin (c), fibroblast growth factor 23 (d), cubilin (e) and AMBP (f) in the urine of patients with Fabry Disease $(n=66)$ and their levels categorized according to disease progression ${ }^{*} p<0.05,{ }^{* *} p<0.01$, ${ }^{* * *} p<0.0001$ (bold data points indicate a patient receiving ERT therapy). The dotted line indicates the control patient upper levels.

Figure 5 Analysis of potential heart disease markers Brain Natriuretic Peptide (a), Glycogen Phosphorylase Brain Form (b), troponin T (c), troponin $I$ (d) and lumican (e) in the urine of patients with Fabry Disease $(n=66)$ and their levels categorized according to disease progression ${ }^{*} p<0.05$, ${ }^{* *} p<0.01,{ }^{* * *} p<0.0001$ (bold data points indicate a patient receiving ERT therapy). The dotted line indicates the control patient upper levels.

Figure 6 Analysis of potential inflammatory markers neutrophil collagenase (a), hemopexin (b), intracellular cell adhesion molecule 1 (c) and $\alpha_{1}$ antitrypsin (d) in the urine of patients with Fabry Disease and their levels categorized according to disease progression ${ }^{*} p<0.05,{ }^{* *} p<0.01$, ${ }^{* * *} p<0.0001$ (bold data points indicate a patient receiving ERT therapy). The dotted line indicates the control patient upper levels.

Supplementary Figure 1 Chromatogram (UPLC-MS/MS) of a targeted and multiplexed proteomic assay developed to quantitate 41 proteins in urine

Supplementary Figure 2 Analysis of urinary albumin indicates there is no significant difference according to symptomatic disease groups.

Supplementary Figure 3 Levels of lyso-gb3 for each patient and grouped according to symptomatic disease. No significant difference is observed in plasma lyso-gb3 levels between the groups according to organ involvement.

Supplementary Figure 4 Correlation matrix of each protein and Plasma lyso-Gb3 levels in the Fabry patient cohort analysed in this study. There is no relationship between the protein levels and lyso-gb3. 
Figure 1
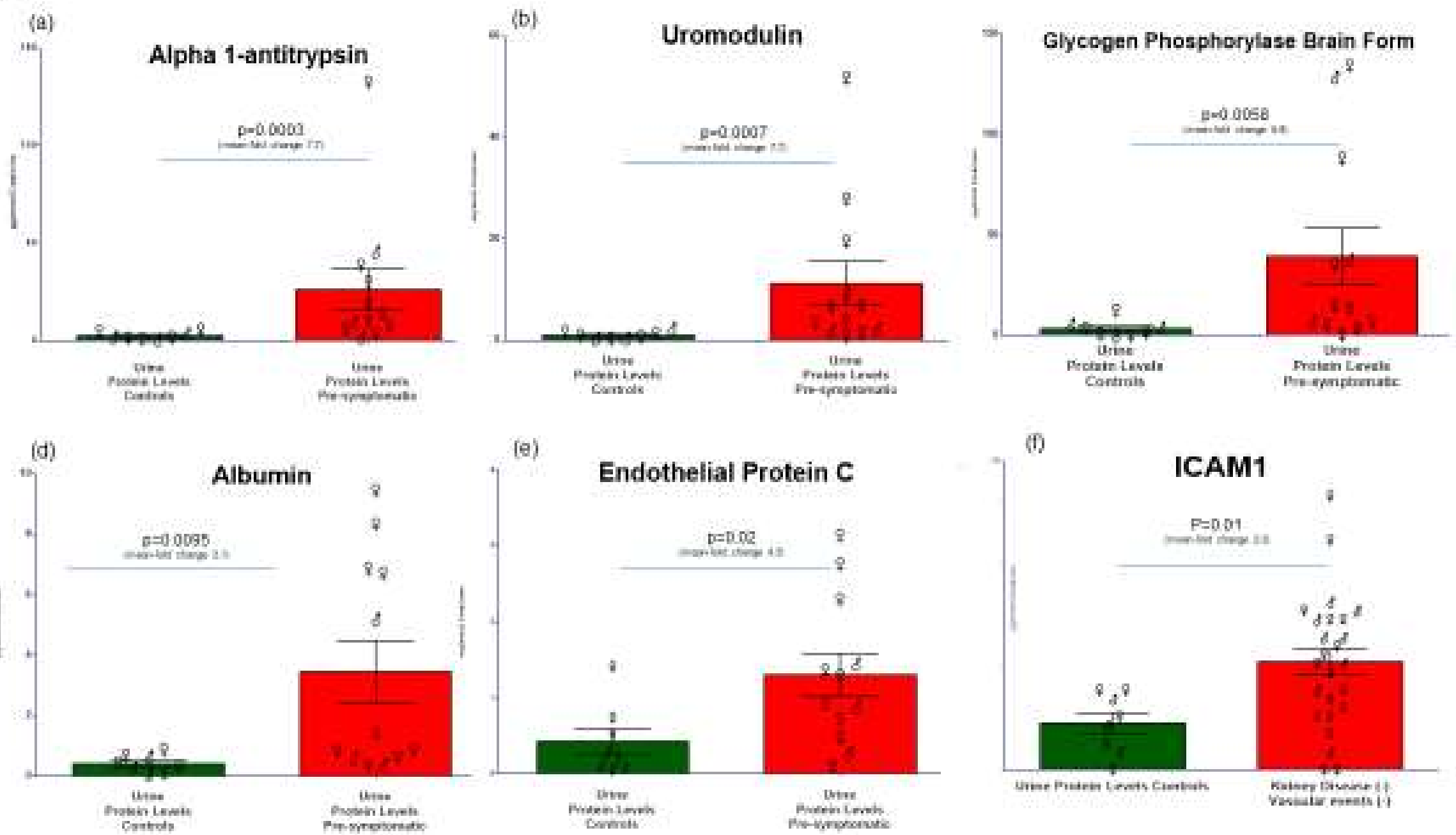
Figure 2

(a)

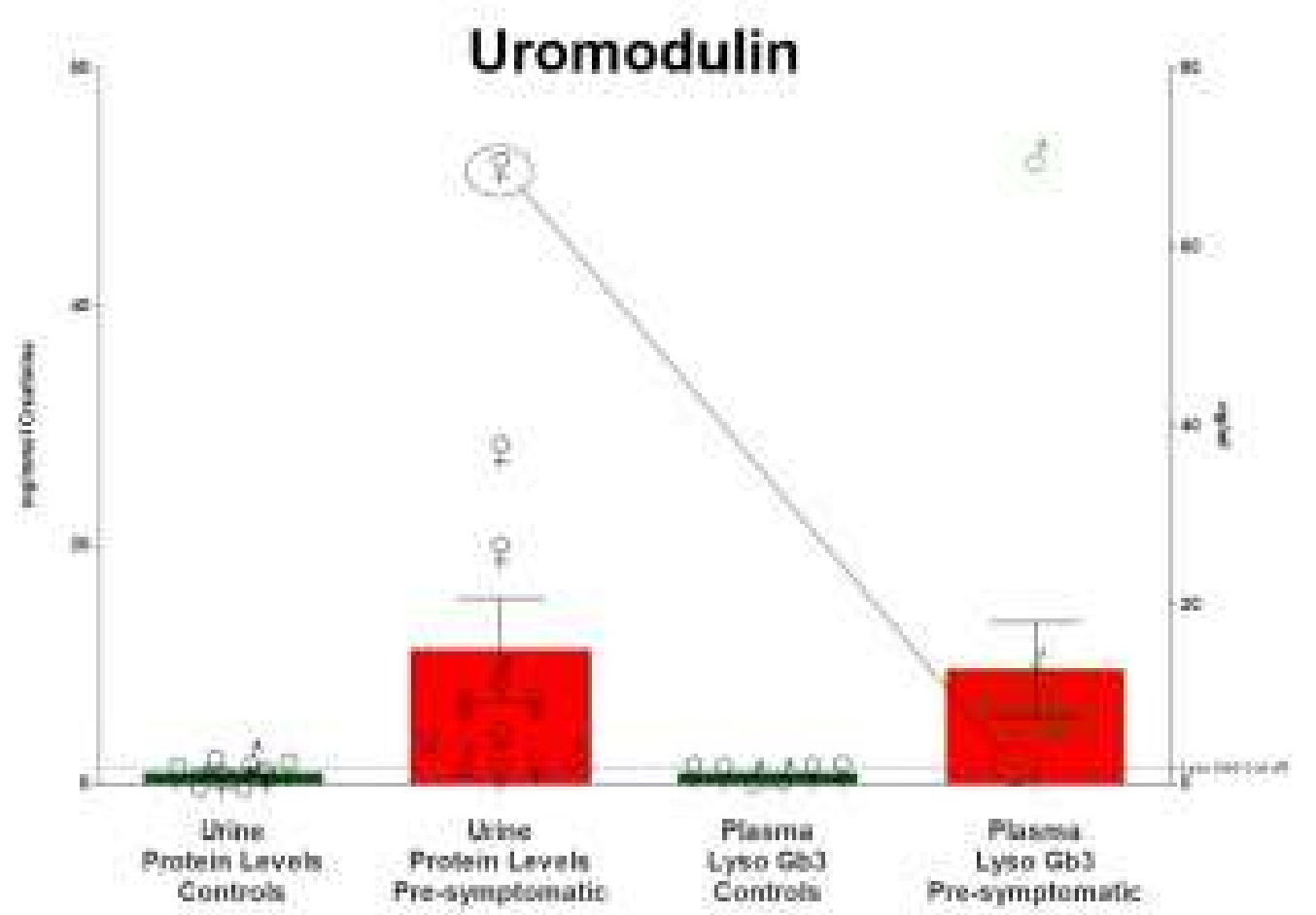

(b)

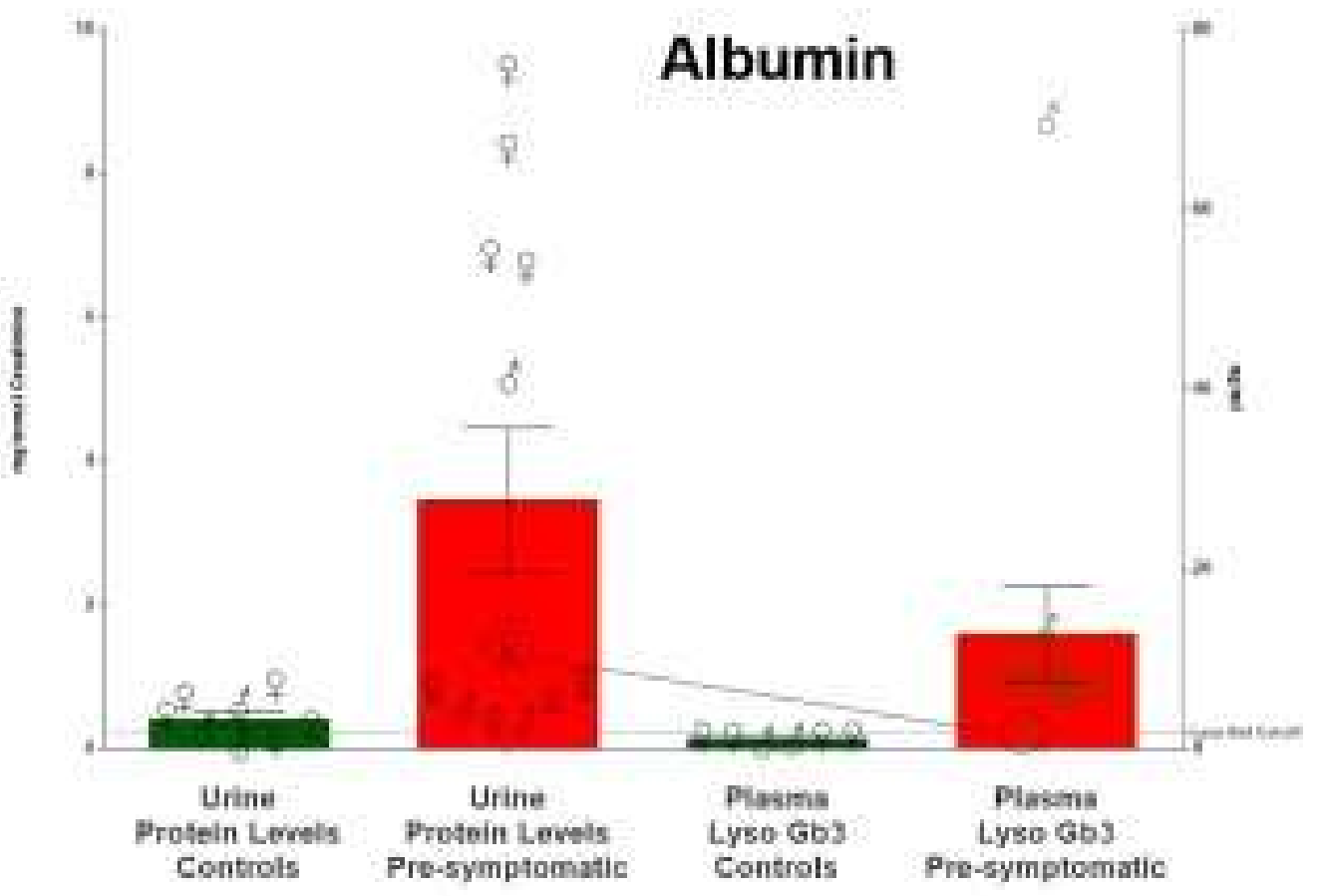




\section{Figure 3}

(1)

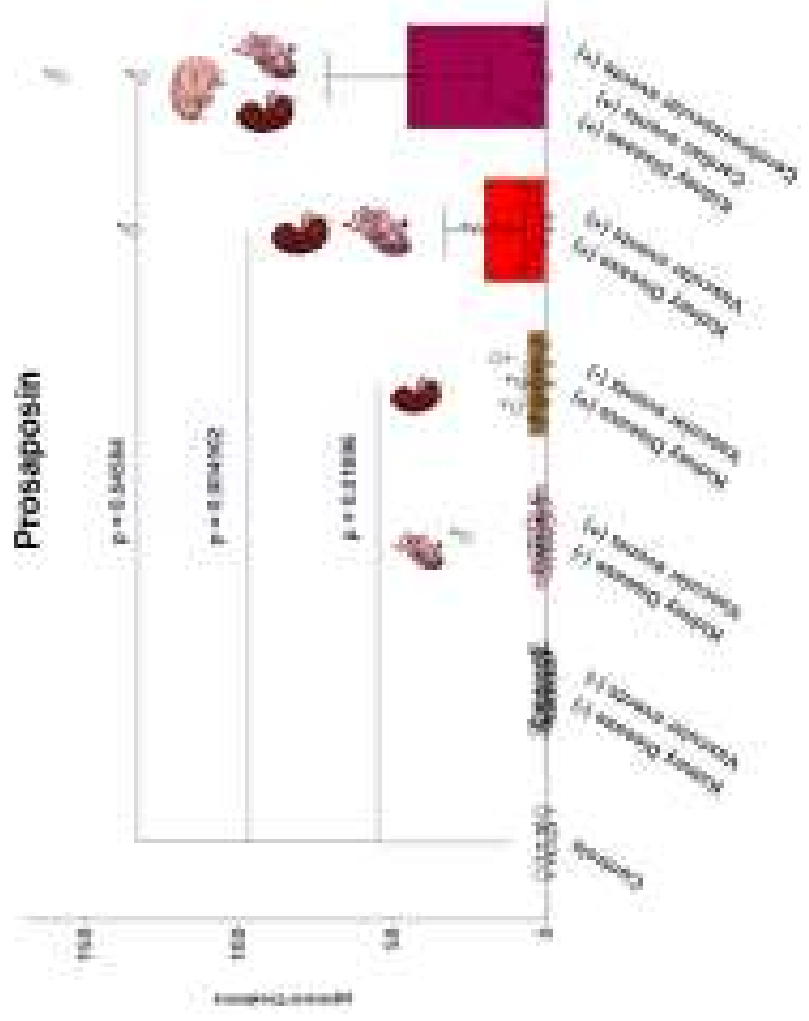



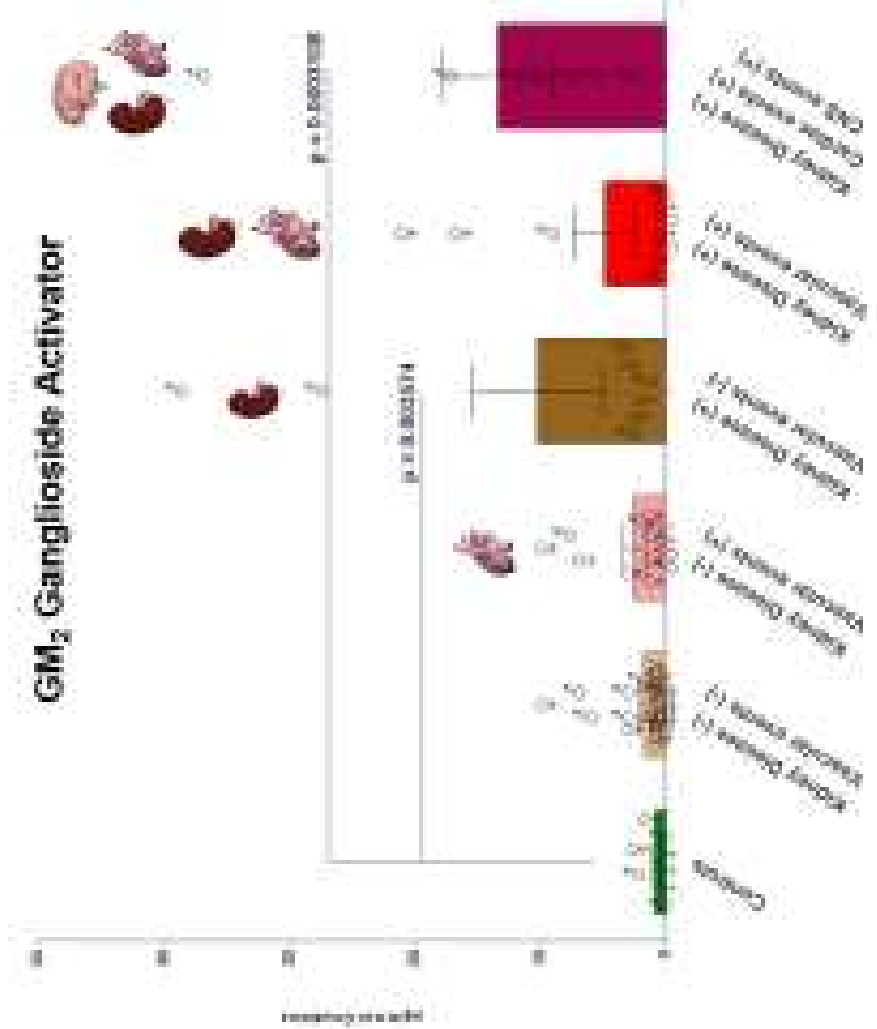

9

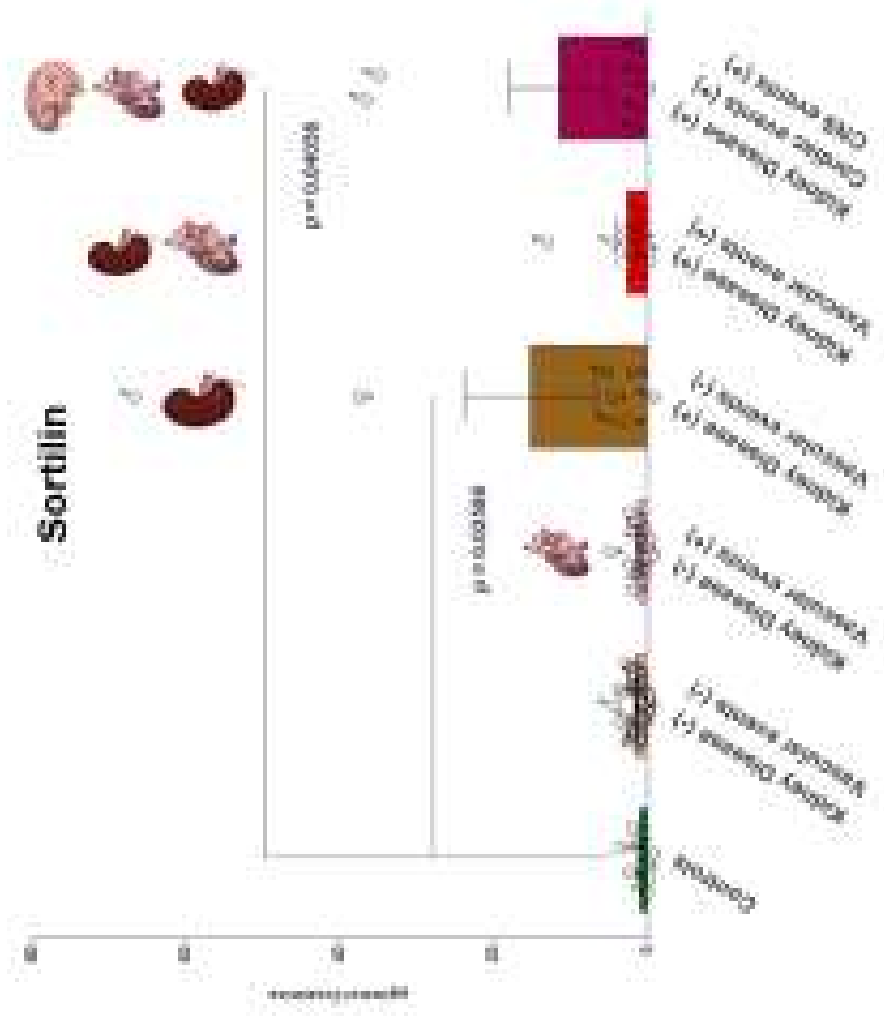


Figure 4
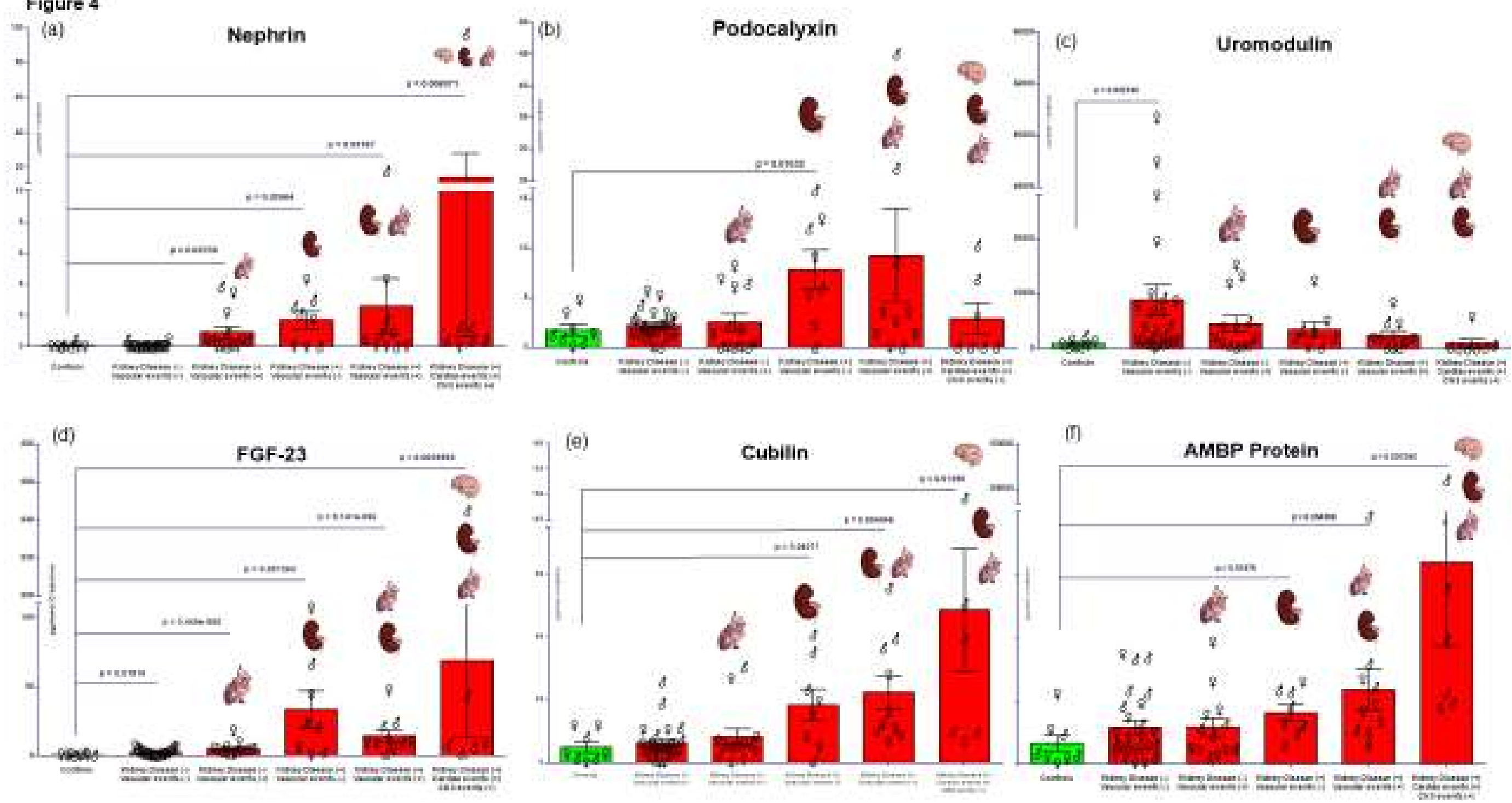


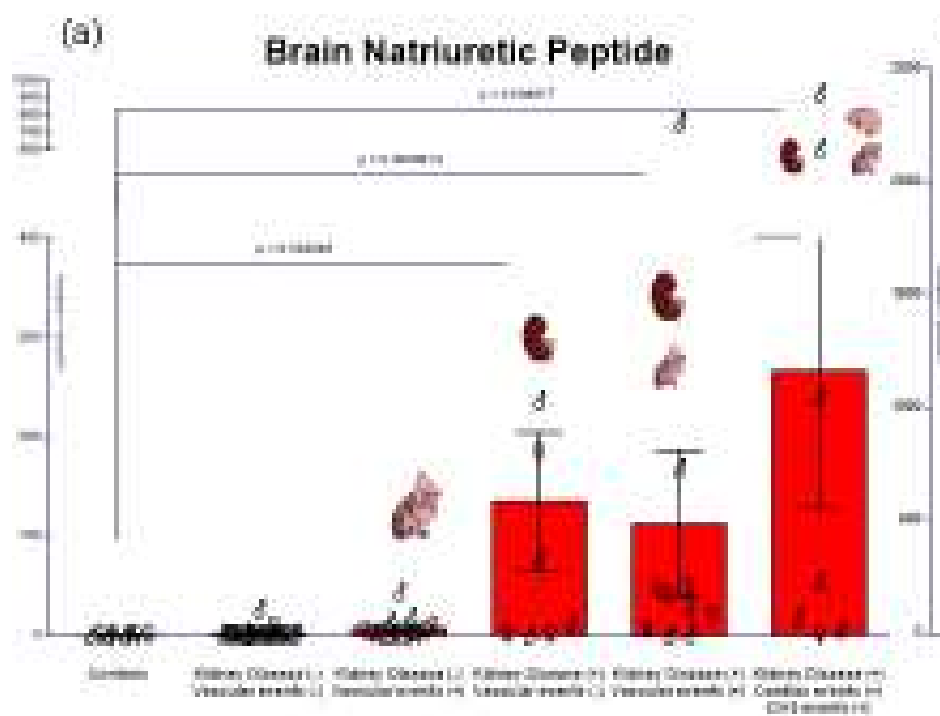

(b) Glyeogen Phosphorylase brain isotom Gycogen Phosphorylase brain lsotom
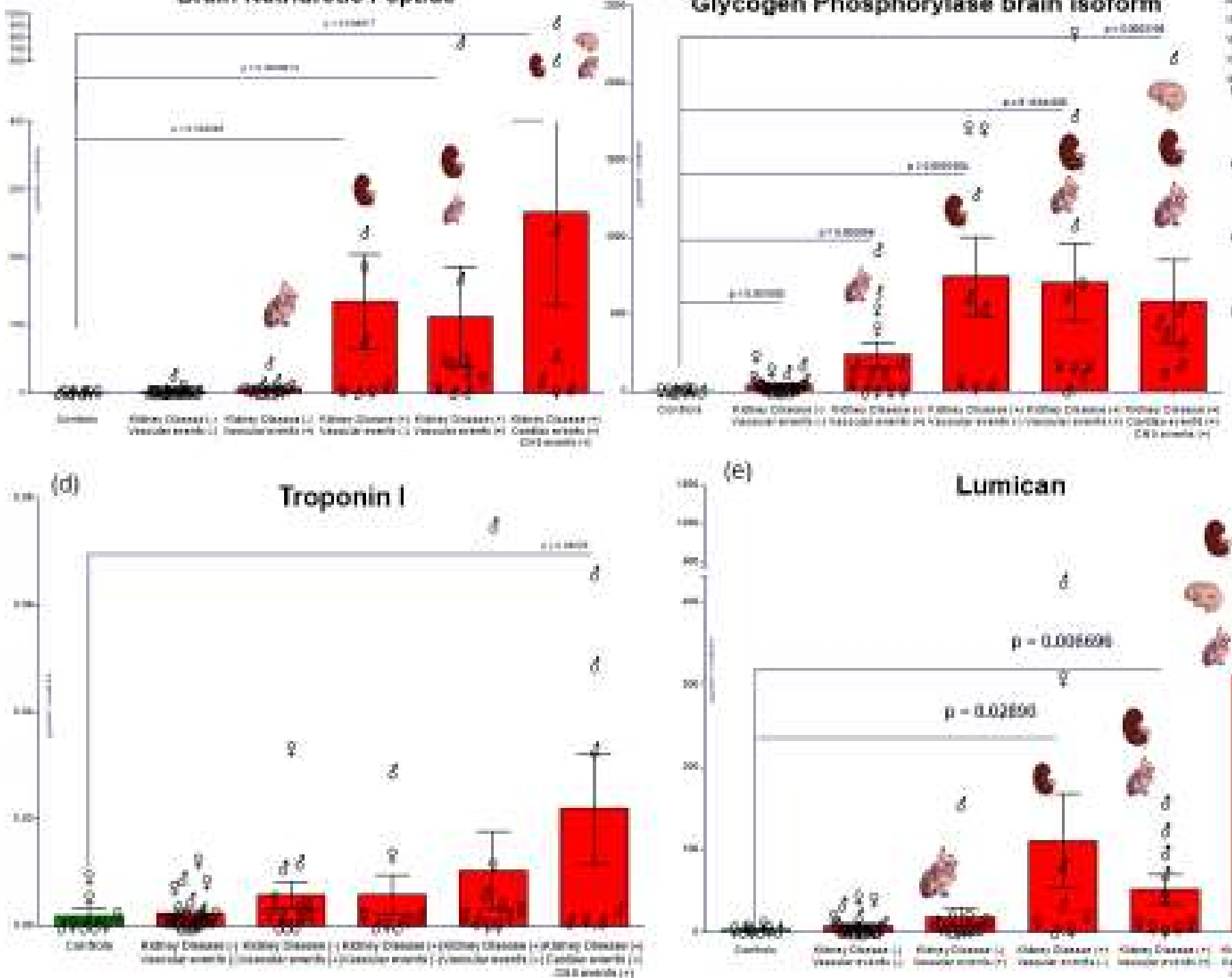

(c)
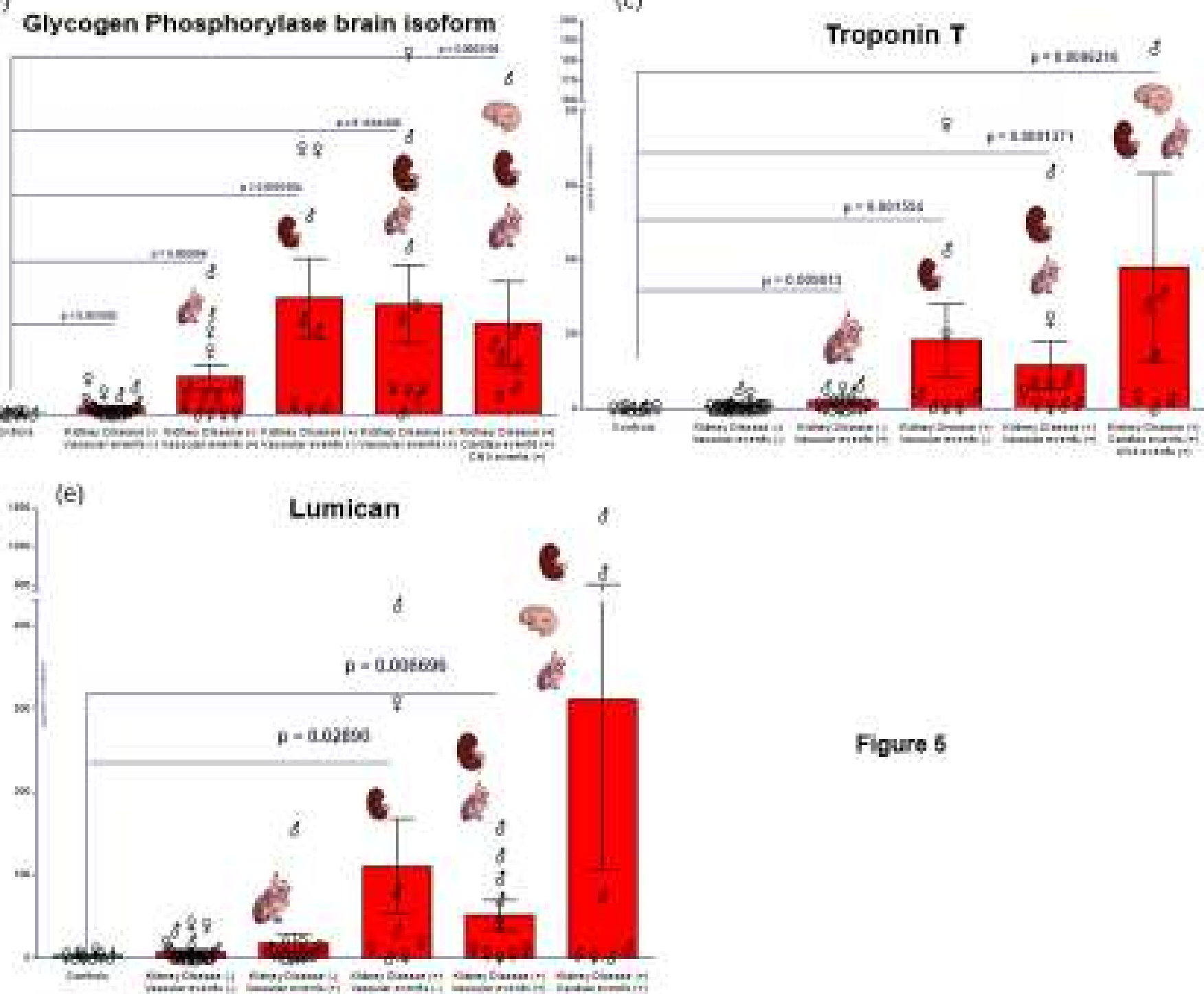

Figure 5 

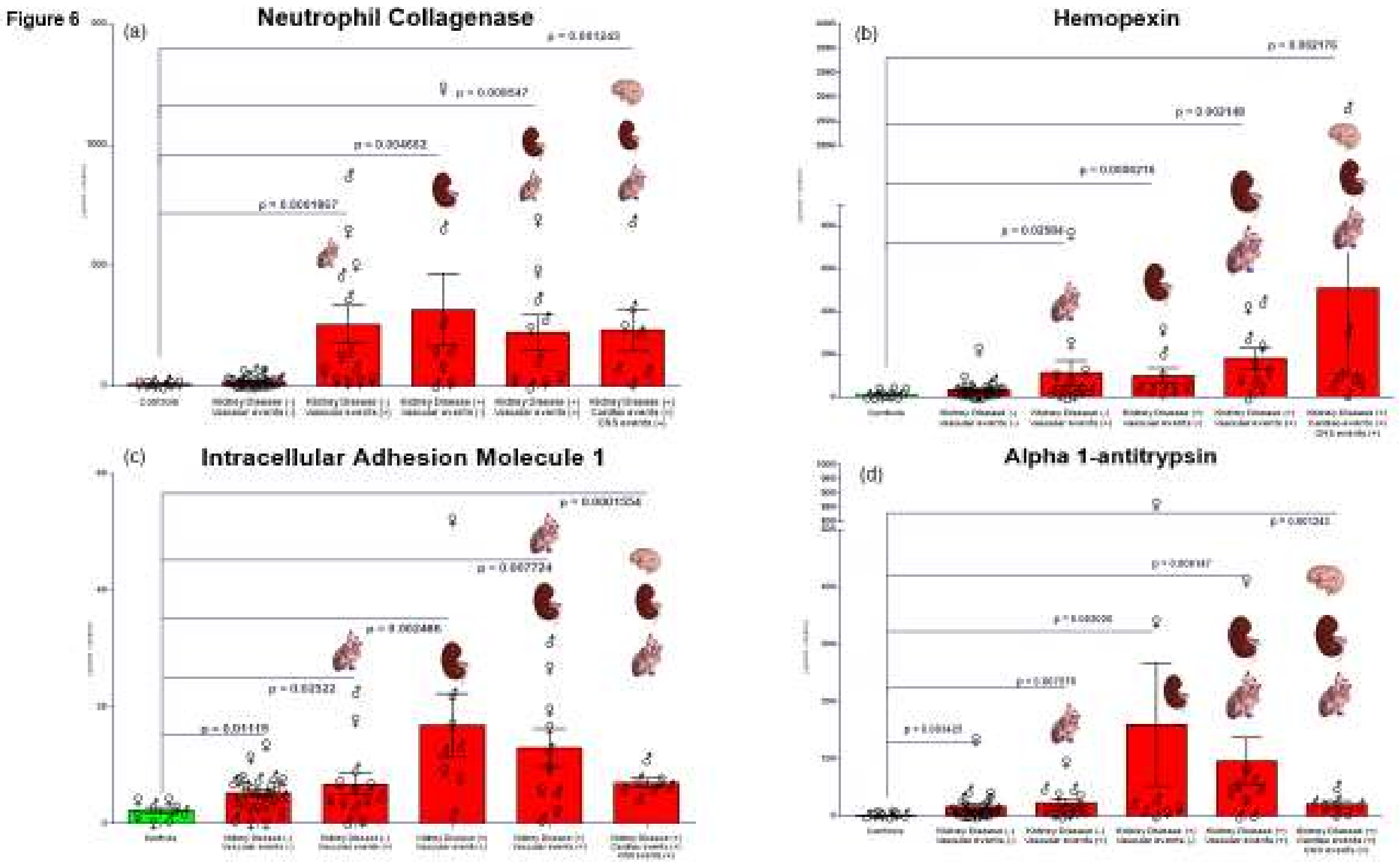